\title{
Socket preservation using eggshell-derived nanohydroxyapatite with platelet-rich fibrin as a barrier membrane: a new technique
}

\author{
Vivekanand Sabanna Kattimani', Krishna Prasad Lingamaneni', Girija Easwaradas Kreedapathi ${ }^{2}$, \\ Kiran Kumar Kattappagari ${ }^{3}$ \\ Departments of ${ }^{1}$ Oral and Maxillofacial Surgery and ${ }^{3}$ Oral and Maxillofacial Pathology, Sibar Institute of Dental Sciences, Guntur, \\ ${ }^{2}$ Department of Physics, Periyar University Salem, Salem, India
}

\begin{abstract}
J Korean Assoc Oral Maxillofac Surg 2019;45:332-342)
Objectives: Socket grafting is vital to prevent bone resorption after tooth extraction. Several techniques to prevent resorption have been described, and various bone graft substitutes have been developed and used with varying success. We conducted this pilot study to evaluate the performance of nanohydroxyapatite (nHA) derived from chicken eggshells in socket preservation.

Materials and Methods: This was a prospective, single center, outcome assessor-blinded evaluation of 23 sockets (11 patients) grafted with nHA and covered with platelet-rich fibrin (PRF) membrane as a barrier. Bone width and radiographic bone density were measured using digital radiographs at 1, 12, and 24 weeks post-procedure. Postoperative histomorphometric and micro-computed tomography (CT) evaluation were performed. The study protocol was approved by the Institutional Ethics Committee.

Results: All patients had uneventful wound healing without graft material displacement or leaching despite partial exposure of the grafted socket. Tissue re-epithelialized with thick gingival biotype ( $>3 \mathrm{~mm}$ ). Width of the bone was maintained and radiographic density increased significantly with a trabecular pattern ( $73.91 \%$ of sockets) within 12 weeks. Histomorphometric analysis showed $56.52 \%$ Grade 3 bone formation and micro-CT analysis revealed newly formed bone with interconnecting trabeculae.

Conclusion: Use of a PRF membrane with nHA resulted in good bone regeneration in sockets. Use of a PRF membrane prevents periosteal-releasing incisions for primary closure, thereby facilitating the preservation of keratinized mucosa and gingival architecture. This technique, which uses eggshellderived nHA and PRF membrane from the patient's own blood, is innovative and is free of disease transfer risks. nHA is a promising economic bone graft substitute for bone regeneration and reconstruction because of the abundant availability of eggshell waste as a raw material.
\end{abstract}

Key words: Bone, Tooth extraction, Socket grafting, Wound healing, Bone regeneration

[paper submitted 2018. 12. 15 / revised 1st 2019. 4. 13, 2nd 2019. 5. 1 / accepted 2019. 5. 12]

\section{Introduction}

Socket grafting is vital to prevent bone resorption ${ }^{1}$. Remodeling of alveolar bone is a continuous process in physiology and pathology, but alveolar bone is essential for the retention of teeth. Extraction of teeth leads to an edentulous space and loss of alveolar bone, function, and aesthetics ${ }^{2-6}$. A systemic

\footnotetext{
Vivekanand Sabanna Kattimani

Department of Oral and Maxillofacial Surgery, Sibar Institute of Dental Sciences, Takkellapadu, Guntur, Andra Pradesh 522509, India

TEL: +91-863-2292249 FAX: +91-863-2292139

E-mail:drvivekanandsk@gmail.com

ORCID: https://orcid.org/0000-0002-9812-7207

(c) This is an open-access article distributed under the terms of the Creative Commons Attribution Non-Commercial License (http://creativecommons.org/ licenses/by-nc/4.0/), which permits unrestricted non-commercial use, distribution, and reproduction in any medium, provided the original work is properly cited. Copyright (C) 2019 The Korean Association of Oral and Maxillofacial Surgeons. All rights reserved.
}

review of dimensional changes of the alveolar bone following extraction showed a mean reduction of $3.8 \mathrm{~mm}$ in width and $1.24 \mathrm{~mm}$ of height in the first six months after extraction? Replacement of missing teeth is essential, but without the support of adequate healthy alveolar bone, teeth replacement is difficult ${ }^{8}$. Socket preservation attempts are therefore made to maintain bone to enable patients to have implant supported prosthesis in the future without the requirement for additional surgical procedures for augmentation ${ }^{1}$.

Socket preservation has been performed using different materials with varying success rates ${ }^{1,9-12}$. Materials used for grafting include autogenous bone, allograft, xenograft, and alloplasts, among others ${ }^{1,9}$. Various membranes have been used for wound closure, including polytetrafluoroethylene and bovine and porcine collagen matrices ${ }^{1}$. Graft materials enhance bone formation by promoting osteoconduction, os- 
teoinduction, and osteogenesis ${ }^{13-16}$. However, no graft material to date is considered ideal. Recently, nanohydroxyapatite derived from chicken eggshell (nHA) was developed ${ }^{17-19}$ and used as an innovative graft material for bone regeneration purposes $^{20-22}$, but none of these previous studies evaluated the performance of nHA and a platelet-rich fibrin (PRF) membrane in socket preservation ${ }^{20-23}$.

We therefore performed this pilot study to evaluate the histomorphometric and radiographic characteristics of bone regeneration when nHA and a PRF membrane were used for alveolar bone preservation and enhancement in the maxilla and mandible of humans to enable implant-supported restoration.
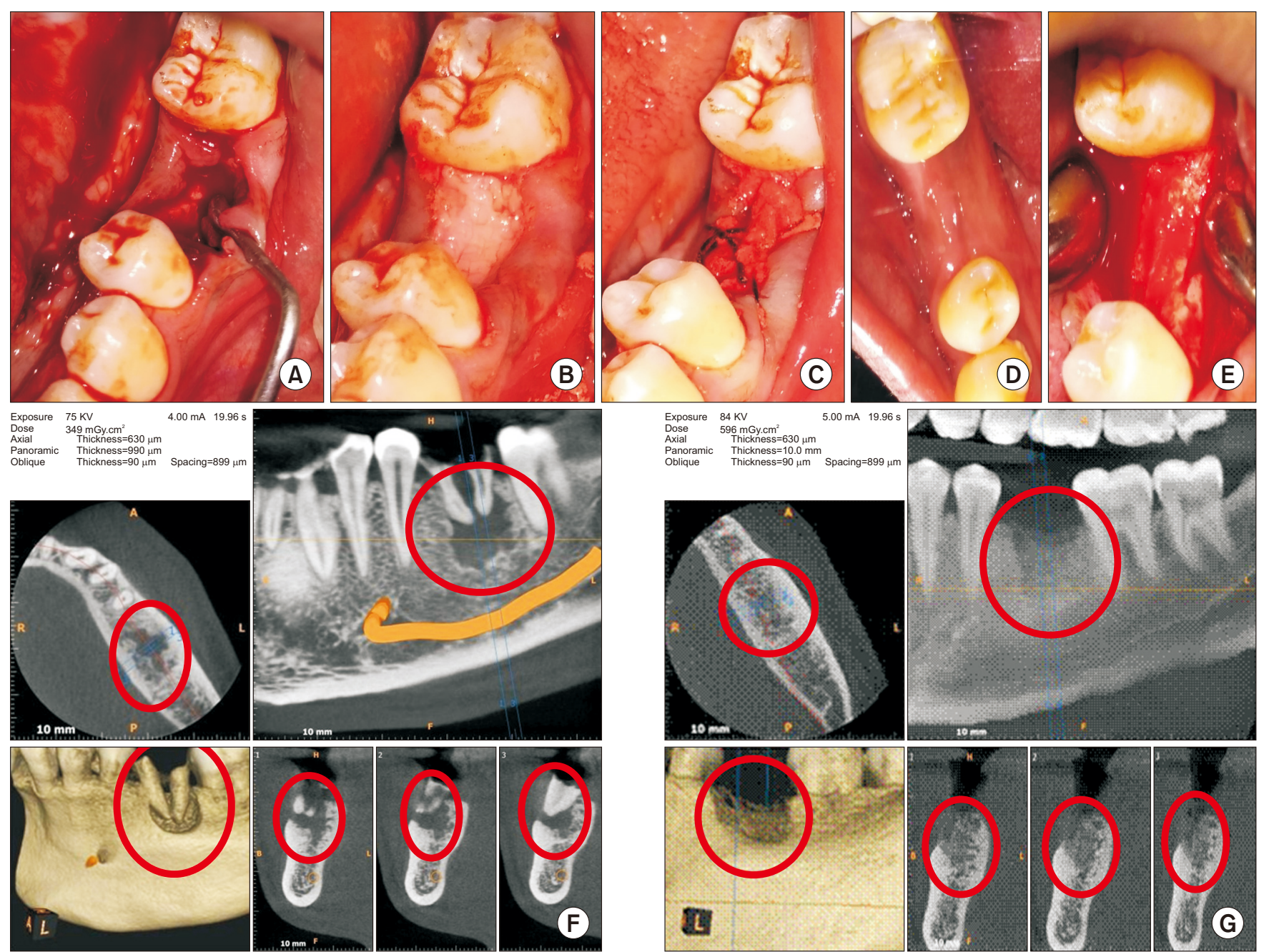

\section{Materials and Methods}

\section{Study design}

A total of 11 (eight male and three female) patients between the ages of 15 to 45 years who presented to the outpatient Department of Oral and Maxillofacial Surgery, Sibar Institute of Dental Sciences (Guntur, India) for extraction and willing to participate in the study protocol were enrolled during the period from June 2017 to June 2018. The Institutional Ethics Committee of Sibar Institute of Dental Sciences approved the study protocol (IEC-16/09/2014) and this study was registered with the Clinical Trials Registry-India (CTRI). Patients and patients' relatives were informed about the study

Fig. 1. A. Bone defect immediately after extraction with curette in place. B. Bone graft material placed till crestal level in the mandibular left first molar socket. C. Wound closure using platelet-rich fibrin membrane and figure of eight suture. D. Well healed wound after two weeks. E. Well-formed bone observed during exposure for implant bed preparation. F. Cone-beam computed tomography pre-extraction view with roots and bone defect marked in red circles. G. Bone formation (red circles) assessed before implant placement.

Vivekanand Sabanna Kattimani et al: Socket preservation using eggshell-derived nanohydroxyapatite with platelet-rich fibrin as a barrier membrane: a new technique. J Korean Assoc Oral Maxillofac Surg 2019 
protocol before enrollment and written informed consent was obtained. This was a prospective, single center, outcome assessor-blinded pilot study.

\section{1) Sample size calculation}

The sample size required for this pilot study was 10 patients but we recruited 11 patients involving one or more sockets per patient depending on the clinical scenario. Sample size was calculated with $80 \%$ power and a $5 \%$ alpha error based on a two-sided test. Total of 23 extraction sockets were assessed among the 11 patients.

2) Inclusion and exclusion criteria for patient selection

Patients with grossly decayed teeth, unrestorable teeth, those willing to undergo implant restoration, and those readily available for periodic recall were enrolled. Pregnant patients, patients with gross tooth mobility due to complete bone loss (more than 3/4 of root length), those in a vulnerable category or with a systemic illness (diabetes, thyroid, hypertension, etc.), and medically compromised patients were excluded from the study protocol to eliminate bias.

\section{3) Materials}

(1) Nanohydroxyapatite

nHA granules used for grafting were synthesized from natural calcium precursors obtained from chicken eggshell waste and produced using a rapid microwave processing technique ${ }^{17,18}$. The nHA obtained in this manner is typical flower-like nanostructured $\mathrm{HA}^{19}$. It is composed of leaf-like flakes extending radially from the center with a width of 100 to $200 \mathrm{~nm}$ and length of 0.5 to $1 \mu \mathrm{m}$. nHA contains $\mathrm{Ca}, \mathrm{P}, \mathrm{O}, \mathrm{C}$, and $\mathrm{Mg}^{18,19}$. Incorporation of $\mathrm{Mg}$ ions into the HA structure is important for the development of artificial bones ${ }^{19}$.

(2) Platelet-rich fibrin membrane

PRF membrane was prepared using autologous blood under standard aseptic precautions. Intravenous blood ( $5 \mathrm{~mL})$ was drawn from the antecubital region using a sterile disposable syringe. Blood was centrifuged using a tabletop centrifuge for 10 minutes at 3,000 rpm immediately after collection. The resultant product comprised acellular plasma as the top layer, a PRF clot in the middle, and red blood cells at the bottom. Successful preparation of PRF depends on speedy blood collection and immediate centrifugation before clotting begins. PRF component was separated and molded into a thin sheet of approximately $1 \times 1 \mathrm{~cm}$ using a sterile, wet piece of gauze.

\section{Operative procedure}

Extraction of the tooth or tooth root was performed atraumatically under local anesthesia. If necessary, curettage was performed (Fig. 1. A) and irrigation was done with Betadine and saline. Socket compression was not performed to prevent the collapse of bony walls. After hemostasis, nHA grafting was performed to the crestal level in all 23 sockets.(Fig. 1. B) PRF membrane was transferred over the grafted socket and tucked between buccal and lingual/palatal gingiva and stabilized using 3-0 black silk suture (figure of eight). The suture passed through the membrane to prevent slippage. PRF membrane prevented leaching of materials and periosteal-releasing incisions for primary closure.(Fig. 1. C) Postoperatively, all patients were administered antibiotics and analgesics for five days as a standard of care. Patients were recalled after 8 days for suture removal.

Implant bed was prepared using a trephine bur $(3 \times 10 \mathrm{~mm})$ followed by a standard sequential drilling protocol for implant placement 24 weeks after grafting. Depending on initial stability, implants were loaded immediately or in a delayed manner using NobelParallel conical connection root form implants made by Nobel Biocare (Kloten, Sweden).

\section{Clinical and radiographic evaluation}

Wound healing was assessed prospectively.(Fig. 1. D) Patients were followed-up for 24 weeks until implant insertion. (Fig. 1. E) Preoperative cone-beam computed tomography (CBCT) revealed bony defects with roots.(Fig. 1. F) Bone width was measured using bone calipers and radiographic bone density was measured using digital intraoral peri-apical radiograph at week 1,12 , and 24 postoperatively. CBCT was obtained for planning and assessment before implant placement.(Fig. 1. G) Digora software (Digora for Windows 2.8.107.458 Network Client, Soredex Orion Corporation, Finland) was used to assess follow-up density changes and bone formation characteristics.(Fig. 2) Radiographic evaluation criteria were modified and adapted for assessment ${ }^{24,25}$. Gridlines in digital IOPA were used as reference lines to assess bone height postoperatively for available bone for implant size selection. The assessor was blinded for the time duration of follow-up during radiographic evaluation.

1) Micro-CT evaluation

During implant placement, a trephine biopsy (taken using a $3 \times 10-\mathrm{mm}$ trephine bur) harvested from the grafted site was 
subjected to histomorphometric and micro-CT evaluation. (Fig. 3) Micro-CT analysis was carried out using a PhoenixV/tome/Xs machine (GE Sensing \& Inspection Technologies GmbH, Wunstorf, Germany).(Fig. 4. A-C) A series of microfocus CT images were acquired as x-ray images while progressively rotating the sample step by step through a $360^{\circ}$ rotation at increments less than $1^{\circ}$ per step.(Fig. 4. D) These projections were used to reconstruct volumetric data. The multiline configuration of the flat panel covered with a movable shield reduced scattering artifacts. A temperature- stabilized DXR500L detector (General Electric, Boston, MA, USA) with 3,072 pixels provided a voxel size of $1.6 \mu \mathrm{m}$ for assessment and allowed scanning of objects with voxel sizes $<2 \mu \mathrm{m}$.

\section{2) Histomorphometric evaluation}

After micro-CT analysis, sections were decalcified using 5\% EDTA. After processing, specimens were embedded in a paraffin wax block and sliced with a microtome to a thickness of $5 \mu \mathrm{m}$ for H\&E staining.(Fig. 5) The core was cut trans-
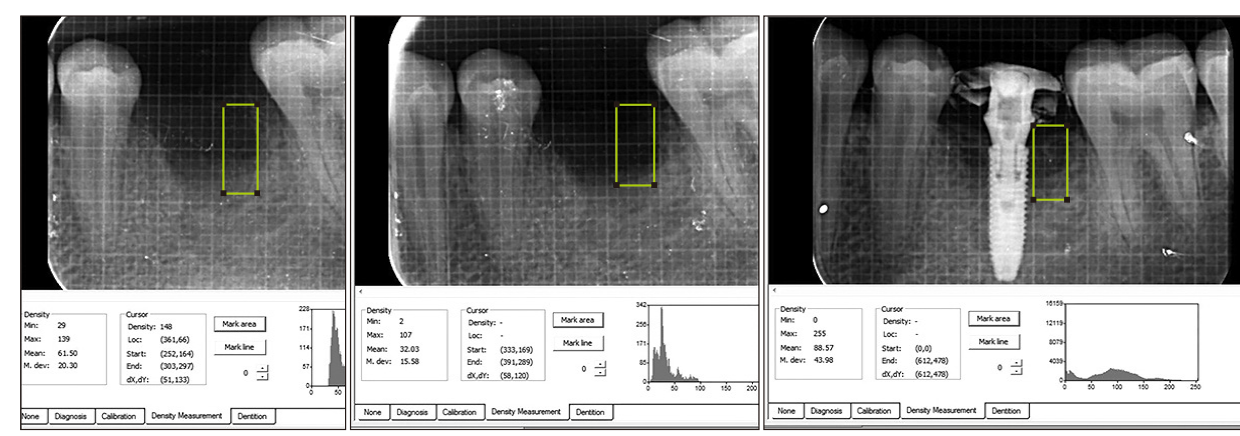

Fig. 2. Intra-oral periapical radiographic images for assessment of bone formation and density using Digora software. Vivekanand Sabanna Kattimani et al: Socket preservation using eggshell-derived nanohydroxyapatite with platelet-rich fibrin as a barrier membrane: a new technique. J Korean Assoc Oral Maxillofac Surg 2019

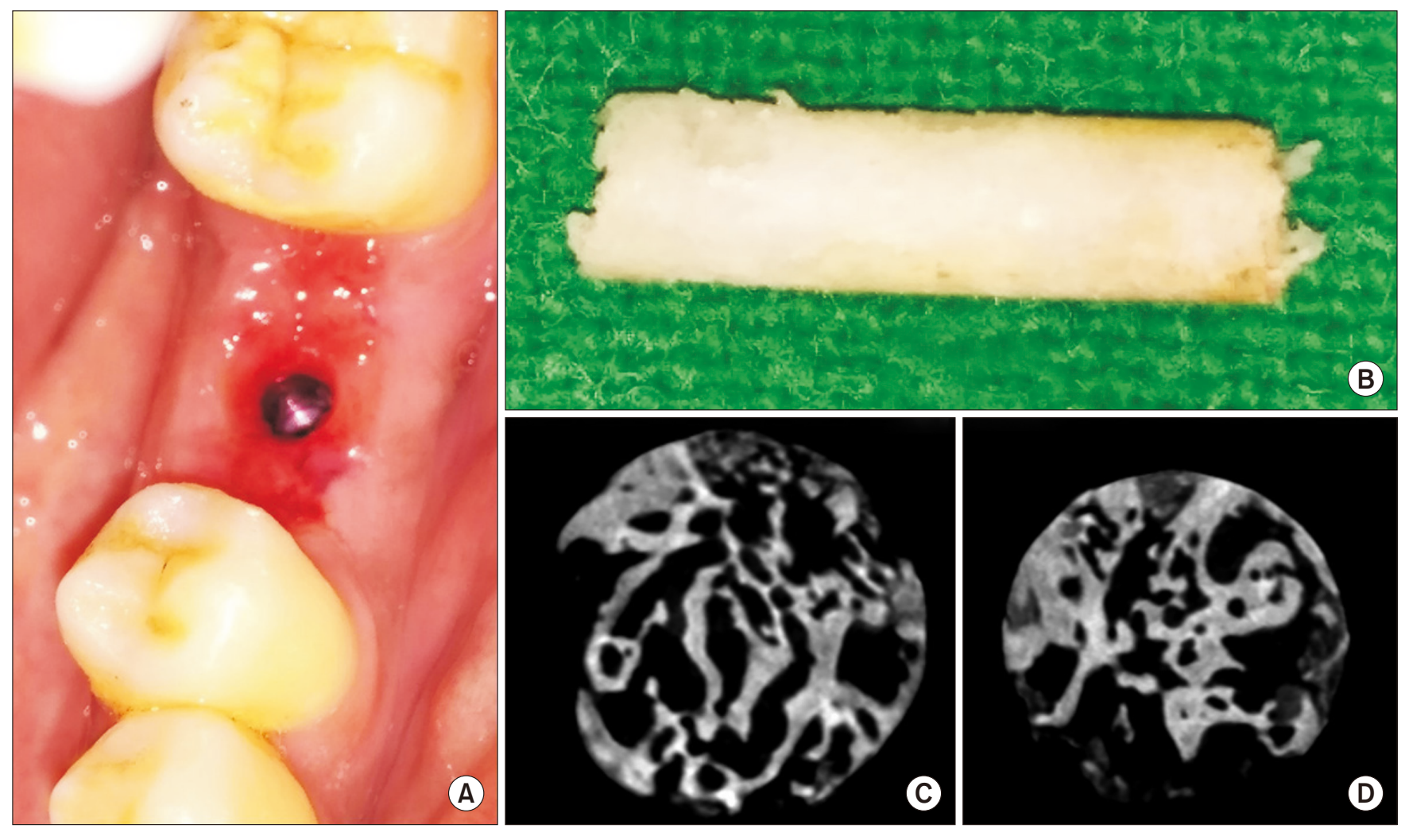

Fig. 3. A. Thick tissue biotype observed after removal of the screw-retained abutment of an immediate-loaded implant following suture removal after 1 week. B. Trephined bone $(3 \times 10 \mathrm{~mm}$ size) harvested from the left mandibular first molar site 24 weeks after grafting (during implant bed preparation) for micro-computed tomography (CT) and histomorphometric analysis. C, D. Micro-CT sections of trephine bone showing intervening trabeculae.

Vivekanand Sabanna Kattimani et al: Socket preservation using eggshell-derived nanohydroxyapatite with platelet-rich fibrin as a barrier membrane: a new technique. J Korean Assoc Oral Maxillofac Surg 2019 


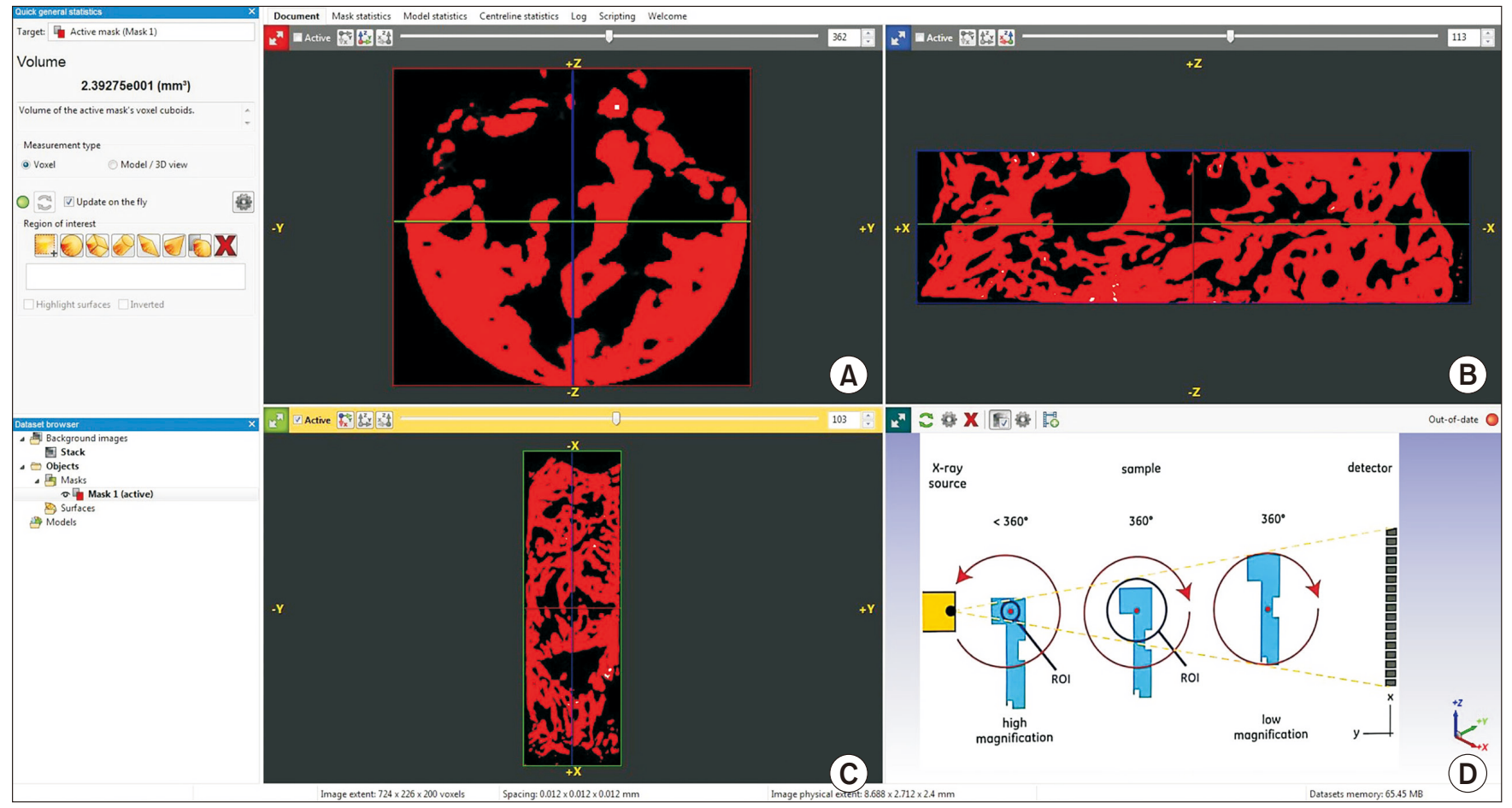

Fig. 4. A-C. Micro-computed tomography (CT) assessment of trephined bone using PhoenixV/tome/Xs machine image analyzing software. D. Schematic presentation of micro-CT image acquisition process for analysis using the PhoenixV/tome/Xs machine.

Vivekanand Sabanna Kattimani et al: Socket preservation using eggshell-derived nanohydroxyapatite with platelet-rich fibrin as a barrier membrane: a new technique. J Korean Assoc Oral Maxillofac Surg 2019
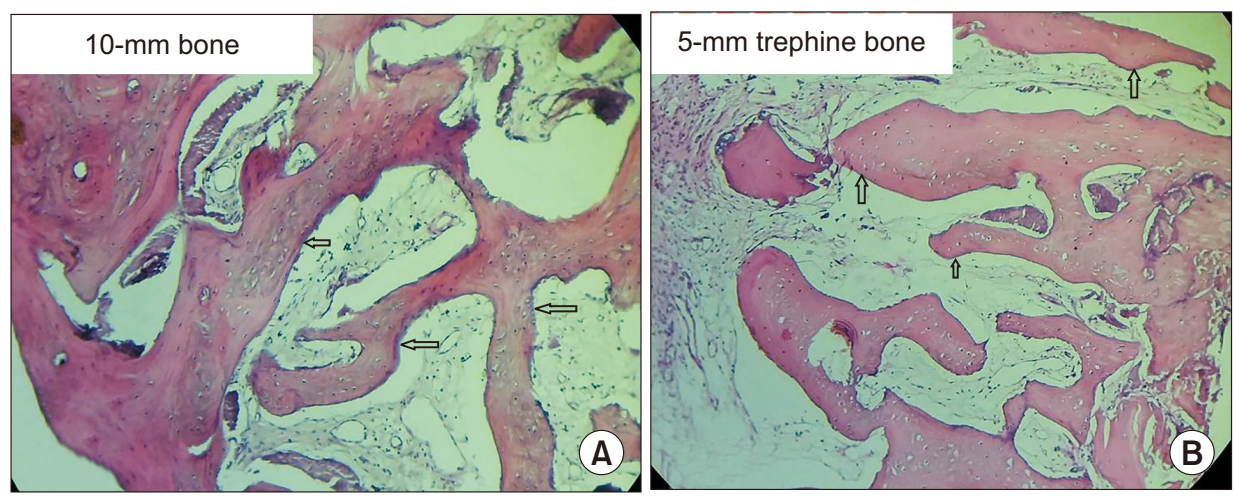

Fig. 5. A. Microphotograph of two $(10$ $\mathrm{mm}$ and $5 \mathrm{~mm}$ ) trephine bone specimens showing intervening trabeculae with a rim of osteoblasts (arrows). B. Osteoid and fibrous connective tissue with plump lacunae with prominent osteocytes was visible (arrows). A, B. H\&E staining $(\times 10)$.

Vivekanand Sabanna Kattimani et al: Socket preservation using eggshell-derived nanohydroxyapatite with platelet-rich fibrin as a barrier membrane: a new technique. J Korean Assoc Oral Maxillofac Surg 2019 versely and interpreted using image analysis software by two oral and maxillofacial pathologists blinded to the procedure. Histology analysis criteria were modified and adopted for evaluation as follows: Grade 0, no bone formation; Grade 1, minimal bone formation; Grade 2, moderate bone formation; Grade 3, abundant bone formation; and Grade 4, exuberant bone formation at the grafted $\operatorname{site}^{26}$.

\section{Statistical analysis}

Results were tabulated using Microsoft Excel 2010 (Microsoft, Redmond, WA, USA). Data were summarized as means and percentages and analyzed by dependent t-tests using IBM SPSS Statistics software (ver. 20.0; IBM, Armonk, NY, USA). Kappa correlation was calculated to assess the degree of observer agreement for radiological assessment.

\section{Results}

\section{Clinical observations}

All patients (23 sockets) had uneventful wound healing. No graft material displacement or leaching was observed, even though the graft material was partially exposed. Tissue 
re-epithelialized entirely within 2 weeks with a thick gingival biotype ( $>3 \mathrm{~mm}$ ). Radiographic analysis showed increased density with a trabecular pattern in $73.91 \%$ of sockets while in the remaining $26.09 \%$ of sockets, a ground glass appearance was observed.(Fig. 6) After 24 weeks, bone fill with well-preserved ridges was observed. Socket wall width was measured at the crestal bone level and was consistent from

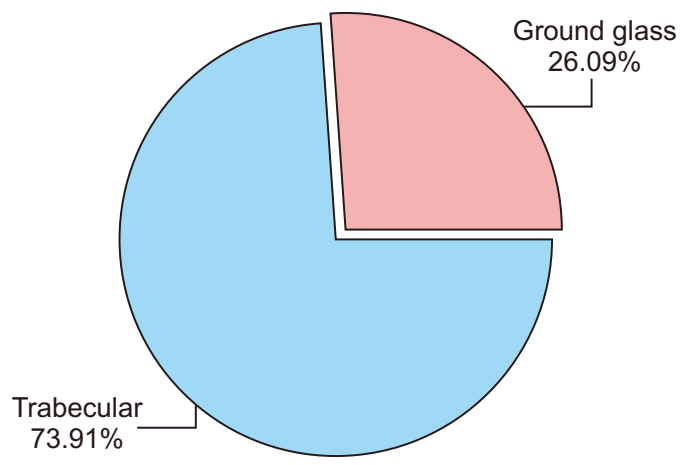

Fig. 6. Pattern of bone regeneration and distribution of these patterns.

Vivekanand Sabanna Kattimani et al: Socket preservation using eggshell-derived nanohydroxyapatite with platelet-rich fibrin as a barrier membrane: a new technique. $J$ Korean Assoc Oral Maxillofac Surg 2019 week 4 to week 24.(Tables 1-3) Graft particles were incorporated into the generated bony tissue and the augmented site was clinically indistinguishable from the original neighboring bony tissue. Many of these sites had successfully placed implants.

\section{Histomorphometric and micro-CT observations}

Micro-CT analysis revealed newly formed bone with interconnecting trabeculae and H\&E staining revealed thin trabeculae of woven bone. Plenty of cellular osteoid in the form of trabeculae with intervening loose relatively dense cellular fibrous tissue was observed. Trabeculae showed a rich anastomosing pattern with numerous enlarged lacunae containing prominent osteocytes. Numerous bundles of collagen fibers were also observed. Osteoid showed eosinophilic areas surrounded by a rim of osteoblasts.(Fig. 5) The graft material was in apposition with the osteoid. No osteoclasts or inflammatory cells were observed. Grade 2 bone formation was observed in $13.04 \%$ of grafted sockets, Grade 3 bone formation in $56.52 \%$ of grafted sockets, and Grade 4 bone formation in $8.70 \%$ of grafted sockets.(Fig. 7 )

Table 1. Socket preservation, changes in alveolar width at different time intervals, pattern of bone regeneration, and histopathology grading 24 weeks after bone grafting

\begin{tabular}{|c|c|c|c|c|c|c|c|c|}
\hline \multirow{2}{*}{ No. } & \multirow{2}{*}{$\begin{array}{l}\text { Tooth No. (FDI) of } \\
\text { socket preservation }\end{array}$} & \multicolumn{2}{|c|}{ Alveolar width $(\mathrm{mm})$} & \multicolumn{3}{|c|}{ Mean density after } & \multirow{2}{*}{$\begin{array}{l}\text { Pattern of bone } \\
\text { regeneration }\end{array}$} & \multirow{2}{*}{$\begin{array}{l}\text { Grade of bone } \\
\text { formation based on } \\
\text { histopathology }\end{array}$} \\
\hline & & Initial & Final & $1 \mathrm{wk}$ & $12 \mathrm{wk}$ & $24 \mathrm{wk}$ & & \\
\hline 1 & 37 & 13 & 12 & 60.60 & 40.32 & 76.34 & Trabecular & 3 \\
\hline 2 & 36 & 14 & 13 & 63.27 & 55.50 & 88.80 & Trabecular & 3 \\
\hline 3 & 11 & 14 & 14 & 54.34 & 34.43 & 70.46 & Ground glass & 3 \\
\hline 4 & 12 & 13 & 13 & 50.12 & 45.10 & 65.27 & Ground glass & 4 \\
\hline 5 & 13 & 15 & 14 & 53.24 & 40.23 & 58.89 & Ground glass & 2 \\
\hline 6 & 14 & 14 & 13 & 50.32 & 43.12 & 65.13 & Ground glass & 4 \\
\hline 7 & 15 & 13 & 12 & 44.45 & 34.10 & 67.73 & Trabecular & 3 \\
\hline 8 & 23 & 15 & 14 & 54.43 & 45.20 & 73.49 & Trabecular & 2 \\
\hline 9 & 24 & 14 & 14 & 50.32 & 40.12 & 75.21 & Trabecular & 3 \\
\hline 10 & 33 & 13 & 13 & 45.56 & 34.34 & 58.12 & Ground glass & 3 \\
\hline 11 & 35 & 13 & 12.5 & 56.75 & 45.65 & 69.15 & Ground glass & 2 \\
\hline 12 & 44 & 13 & 12.5 & 45.43 & 34.43 & 72.14 & Trabecular & 3 \\
\hline 13 & 45 & 14 & 13 & 45.21 & 32.54 & 73.16 & Trabecular & 3 \\
\hline 14 & 36 & 14.5 & 14.5 & 50.68 & 45.00 & 69.76 & Trabecular & 3 \\
\hline 15 & 21 & 14 & 13.5 & 54.45 & 34.80 & 68.23 & Trabecular & 3 \\
\hline 16 & 36 & 14.5 & 14 & 50.89 & 29.01 & 75.20 & Trabecular & 3 \\
\hline 17 & 36 & 15 & 14 & 59.43 & 36.03 & 76.08 & Trabecular & 3 \\
\hline 18 & 36 & 14 & 14 & 66.34 & 35.04 & 79.05 & Trabecular & - \\
\hline 19 & 38 & 15 & 14.5 & 63.12 & 60.06 & 80.03 & Trabecular & - \\
\hline 20 & 14 & 14 & 13.5 & 64.34 & 58.09 & 76.83 & Trabecular & - \\
\hline 21 & 15 & 13 & 13 & 44.65 & 35.07 & 69.01 & Trabecular & 3 \\
\hline 22 & 47 & 15 & 14 & 56.87 & 43.05 & 70.01 & Trabecular & - \\
\hline 23 & 48 & 16 & 15 & 68.54 & 46.03 & 74.01 & Trabecular & - \\
\hline
\end{tabular}

(FDI: Fédération Dentaire Internationale; FDI World Dental Federation)

${ }^{1}$ Histologic analysis graded on the following 5-point scale: 1) Grade 0, no bone formation; 2) Grade 1, minimal bone formation; 3) Grade 2 , moderate bone formation; 4) Grade 3, abundant bone formation; 5) Grade 4, exuberant bone formation.

Vivekanand Sabanna Kattimani et al: Socket preservation using eggshell-derived nanohydroxyapatite with platelet-rich fibrin as a barrier membrane: a new technique. J Korean Assoc Oral Maxillofac Surg 2019 
Table 2. Comparison of initial and final alveolar width $(\mathrm{mm})$ scores by dependent t-test

\begin{tabular}{cccccccc}
\hline Time points & Mean & SD & Mean Diff. & SD Diff. & \% of change & Paired t & $P$-value \\
\hline Initial & 14.04 & 0.86 & & & & & \\
Final & 13.48 & 0.80 & 0.57 & 0.43 & 4.02 & 6.2395 & $0.0001 *$ \\
\hline
\end{tabular}

(SD: standard deviation, Diff.: difference)

$* P<0.05$.

Vivekanand Sabanna Kattimani et al: Socket preservation using eggshell-derived nanohydroxyapatite with platelet-rich fibrin as a barrier membrane: a new technique. J Korean Assoc Oral Maxillofac Surg 2019

Table 3. Comparison of density scores 1 week, 12 weeks, and 24 weeks by dependent t-test

\begin{tabular}{|c|c|c|c|c|c|c|c|}
\hline Time points & Mean & $\mathrm{SD}$ & Mean Diff. & SD Diff. & $\%$ of change & Paired t & $P$-value \\
\hline 1st wk & 54.49 & 7.35 & & & & & \\
\hline 12th wk & 41.19 & 8.26 & 13.31 & 7.17 & 24.42 & 8.9052 & $0.0001 *$ \\
\hline $1 \mathrm{st} w \mathrm{k}$ & 54.49 & 7.35 & & & & & \\
\hline 24th wk & 71.83 & 6.74 & -17.34 & 6.32 & -31.81 & -13.1497 & $0.0001 *$ \\
\hline 12th wk & 41.19 & 8.26 & & & & & \\
\hline 24th wk & 71.83 & 6.74 & -30.65 & 8.29 & -74.41 & -17.7222 & $0.0001 *$ \\
\hline
\end{tabular}

(SD: standard deviation, Diff.: difference)

$* P<0.05$.

Vivekanand Sabanna Kattimani et al: Socket preservation using eggshell-derived nanohydroxyapatite with platelet-rich fibrin as a barrier membrane: a new technique. J Korean Assoc Oral Maxillofac Surg 2019

\section{Discussion}

\section{General aspects}

Socket preservation is essential for maintaining adequate alveolar bone height and width and enhancing bone regeneration. Alveolar bone is required to support the growth of thick soft tissue. Various materials and techniques have been used for socket preservation ${ }^{1,27}$. Selection of a socket preservation technique and graft material remains the choice of the clinician. Many factors such as the pattern of resorption (resorbable or non-resorbable), graft material origin (bovine, synthetic, coral, eggshell), material availability, economic factors, and understanding of the evidence for regeneration influence the choice of graft material ${ }^{14-16}$.

The disadvantages associated with harvesting autogenous grafts have prompted research into novel materials for graft$\mathrm{ing}^{14}$. An increasing number of synthetic alternatives are becoming available ${ }^{16}$. Improvements in technology have facilitated the synthesis of hydroxyapatite graft particles that are osteoinductive ${ }^{28-30}$.

Synthetic HA is a calcium phosphate material that can have different densities, structures, and surface chemistries depending on its exact composition. These characteristics are responsible for the bone bonding properties, turnover rate, and longevity of the material in situ ${ }^{18}$. The efficacy of various HA materials in long-term ridge preservation has been proven. However, HA used in socket preservation should

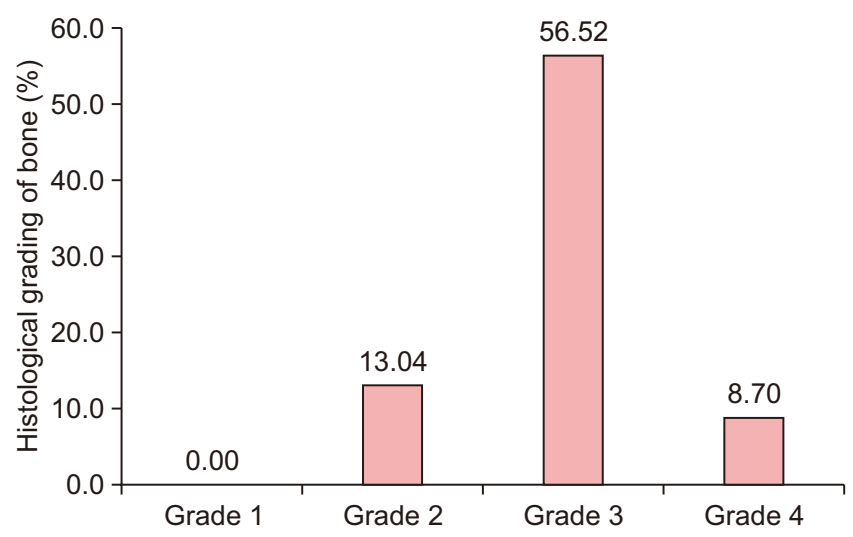

Fig. 7. Histological grade of bone regeneration and distribution of these grades.

Vivekanand Sabanna Kattimani et al: Socket preservation using eggshell-derived nanohydroxyapatite with platelet-rich fibrin as a barrier membrane: a new technique. $J$ Korean Assoc Oral Maxillofac Surg 2019

preserve existing bone and modulate early bone regeneration to facilitate implant osseointegration ${ }^{31,32}$. Synthetic nHA does not require a donor site, and raw material is available in unlimited quantities ${ }^{26,33}$. Control of particle size and interparticulate spaces to mimic those of natural bone is possible during manufacturing ${ }^{17-19}$.

\section{Nanohydroxyapatite derived from chicken eggshell}

We used nHA derived from chicken eggshell in the present study. Nanosynthetic HA has shown promising results for socket preservation, and our findings further support the util- 
ity of nanosynthetic HA for this purpose $\mathrm{e}^{34}$. Histology sections from superficial to deeper cuts showed a consistent increase in newly formed bone with a decrease in the connective tissue area fraction. Histomorphometric findings indicated moderate to abundant bone formation within 24 weeks. Moreover, lower amounts of residual graft particles were observed, similar to the study of Goetz et al. ${ }^{35}$. Goetz et al. ${ }^{35}$ used NanoBone synthetic material for socket filling and reported nearly complete ossification with small residues of material after 4 months follow-up. NanoBone was osteoconductive based on its ability to promote bony ingrowth and initiate integration with newly formed bone. Thus total incorporation of generated bony tissue and mineral particles was achieved ${ }^{34,35}$. New tissue formation in the healed socket was observed in approximately $75 \%$ of all examined specimens. Ultimately, the graft material should induce new bone formation ${ }^{34}$. New bone formation was evident in all samples and augmented sockets had an adequate morphology and density.

Preliminary experimental studies have also evaluated nanosized ceramics as a promising class of graft substitute materials because of their osteo-integrative properties ${ }^{36}$. In these studies, nHA paste was used with or without an additional barrier membrane ${ }^{36}$. This paste promoted wound healing in the extraction sockets. Nanoparticles can hypothetically reduce the inflammatory response and support active osseous organization $^{36,37}$

The nHA used in this study meets the characteristics of an ideal graft material noted by Gross ${ }^{31}$ in 1997. The material is available in granular/nanopowder form, which is easy to handle, and is not prone to infection. In this study, the grafted area was exposed to the oral cavity because we did not use primary closure or a barrier membrane other than a PRF membrane to prevent initial leaching of the material until it became cohesive with a blood clot. nHA is hydrophilic and sticks to the socket wall and settles down in the blood soon after filling. There is no possibility of disease transfer because the material is derived from chicken eggshell. Histology clearly showed the development of bone reminiscent of native bone. Mobility of the tooth has been shown to be maintained when this material is grafted into large cystic defects ${ }^{23}$, and it also supports implant prostheses successfully. Similar findings have been reported for synthetic grafts ${ }^{38}$ with clinically successful implants observed after five years ${ }^{38}$. Furthermore, bone density and volume have been shown to increase over time $e^{38,39}$. As noted by Boyne ${ }^{40}$, synthetics act as a natural barrier for epithelial downgrowth into the socket. The ability to maintain the height and width of alveolar bone and soft tissue anatomy has distinct functional and esthetic advantag$\mathrm{es}^{33,40}$. We observed a significant increase in density from the week 1 to week $12(P<0.05)$. Minimal bone loss $(0.57 \pm 0.43$ $\mathrm{mm}$ ) was observed over 24 weeks compared to baseline, consistent with the literature ${ }^{1}$. Alveolar bone preservation and replacement therapy can be achieved using nHA without a collagen barrier membrane. nHA is therefore a promising graft material that can be produced in an environmentallyfriendly manner ${ }^{17,19}$.

\section{Socket preservation technique and barrier membrane}

We used nHA for socket preservation ${ }^{23}$. No cases of leaching or displacement of the graft material were observed and no periosteal-releasing incisions occurred. Use of an invasive method is recommended against at this point as any procedure that requires primary intention healing with the advancement of flaps can result in an increased inflammatory response and a decrease in vestibular depth, creating an unaesthetic scar ${ }^{41}$. The study by Fickl et al. ${ }^{42}$ showed that flap elevation results in the loss of more bone. This is because rupture of the periosteum and connective tissue insertion consequently reduces blood flow, causing lysis of osteocytes and necrosis of mineralized tissue ${ }^{42}$. This necrotic bone will gradually be removed through resorption by osteoclasts present in the periosteum ${ }^{43,44}$. We overcame this problem of bone loss by not elevating the flap. Elevation also negatively affects the esthetics of the ridge and papilla by altering the mucogingival line position ${ }^{2}$. We used PRF membrane to cover the graft material during the initial period; we consider this an essential step for successful grafting with the existing attached gingiva and future success with implant restoration.

Many current socket preservation techniques use a barrier membrane for primary closure, but there are often difficulties in adoption of the membrane, and a second surgical procedure is required to remove the membrane ${ }^{45}$. In general, membrane use is time-consuming and expensive ${ }^{45-47}$. Murphy ${ }^{45,46}$ reported exposure of non-resorbable membrane in $87 \%$ of cases, leading to infection, swelling, sloughing, and recession. We did not encounter these complications using PRF membrane to cover the graft material. Synthetic grafts do not require a second surgical procedure, nor do they elicit an inflammatory reaction, which is usually seen with a barrier membrane ${ }^{47}$. Formation of dense lamellar bone at the grafted area has been observed ${ }^{20}$. Socket grafting eliminates or reduces the incidence of dry socket ${ }^{33}$; consistent with this, no cases of dry socket were encountered in our study. Moreover, inva- 
sive procedures like guided bone regeneration and sinus floor elevation are not needed when socket grafting is adopted and planned properly before extraction ${ }^{41}$.

The PRF membrane in our study acted as a cover for graft material and as a barrier membrane for guided bone regeneration. The effect of PRF as a barrier membrane for bone formation and soft tissue healing may be limited, but PRF and nHA may interact additively or synergistically. The usefulness of PRF in the maxillofacial area is expanding ${ }^{48}$. Our study design did not allow us to distinguish between the effects of nHA and PRF, although histomorphometry analysis at the bottom of the socket and middle of the socket showed similar bone formation characteristics as that in the crestal area. In previous studies, nHA alone enhanced bone formation in cystic defects ${ }^{23,49}$. Even after disintegration of the PRF membrane, material leaching was not observed. Alveolar bone loss occurs if socket preservation techniques are not employed ${ }^{1}$. We therefore did not include a control group in this preliminary study as this study is part of an ongoing study protocol to delineate the histomorphometric and microCT characteristics of bone formation in relation to radiography; previous studies have used only radiography (IOPA and CBCT/CT scan) for assessment of cystic defect grafting ${ }^{23,49}$.

Grafting prevents hematoma and infection. Manipulation of graft material is easy because of the hydrophilic nature of nHA. It can be used as putty when mixed with a drop of saline or native blood or serum from the PRF test-tube. Its ease of use, absence of disease transfer risk, ready availability in large quantities, and environment-friendly production make nHA an economic graft substitute material for enhancement of bone regeneration and reconstruction. Application of $\mathrm{nHA}$ in grafted sockets supports implant restoration, indicating osseointegration.

\section{Future research work}

Advances in tissue engineering techniques are likely to provide novel biomaterials for everyday clinical use ${ }^{41}$. Longterm follow-up data are mandatory to evaluate the presence of grafted particles that could eventually interfere with the longevity of the implant. We are currently performing a study to further elucidate the histochemical and morphometric nature of the bone formed, as well as assess the resorption kinetics of nHA. The results of the current pilot study suggest that multicenter randomized controlled clinical trials with long-term implant restoration follow-up are warranted. Various grafting materials are available for purchase. Cost varies according to the manufacturer and depends on many factors such as the raw material source and processing technique involved $^{14,16,31,32}$. Comparison of the costs of grafting is beyond the scope of this study.

Limitations of this pilot study are our small sample size and the fact that socket morphology, width, and presence of buccal/lingual cortical walls were not taken into account in our analyses.

\section{Conclusion}

Sockets preserved with nHA showed good bone regeneration. Use of nHA and a PRF membrane as a barrier can assist the healing process and provide better bone quality while preserving alveolar bone. Micro-CT provides good insight into bone healing and bone quality in three dimensions compared to conventional histopathology. The technique described here does not require primary closure, thereby facilitating the preservation of keratinized mucosa and gingival architecture. nHA derived from chicken eggshell is a promising novel bone graft substitute.

\section{ORCID}

Vivekanand Sabanna Kattimani, https://orcid.org/00000002-9812-7207

Krishna Prasad Lingamaneni, https://orcid.org/0000-00020098-2900

Girija Easwaradas Kreedapathi, https://orcid.org/00000002-2529-0062

Kiran Kumar Kattappagari, https://orcid.org/0000-00016614-6617

\section{Authors' Contributions}

V.S.K. designed the study and K.P.L. mentored the study. V.S.K. collected data and wrote the manuscript. K.K.K. and G.E.K. performed the review and assisted for the study. K.K.K. participated in the study assessment and helped to draft the histological assessment part of the manuscript. All authors read and approved the final manuscript.

\section{Acknowledgements}

We would like to thank our colleagues in the Department of Oral and Maxillofacial Radiology and Oral and Maxillofacial Pathology for their support during execution of this 
study protocol as well as the Department of Physics, Periyar University, Salem for their collaboration. The Department of Sciences and Technology, Government of India provided funding for synthesis of nHA under the Waste Management Technology Development and Transfer division. We would also like to thank Prof. Krishnan Balasubramanian and Mr. Harikrishnan Ravichandran of the Center for Nondestructive Evaluation, Indian Institute of Technology Madras, Chennai, Tamil Nadu for the micro-CT evaluation of the specimens. Finally, we would like to thank Dr. Javali for his guidance with statistical analysis.

This study was supported by Department of Science and Technology, Government of India (grant No. DST/TSG/ $\mathrm{WM} / 2015 / 576 / \mathrm{G})$. The study protocol is registered with the Clinical Trials Registry-India (CTRI; CTRI/2014/12/005340).

\section{Ethics Approval and Consent to Participate}

The study protocol was approved by the Institutional Ethics Committee of Sibar Institute of Dental Sciences (IEC$16 / 09 / 2014$ ), and the written informed consent was obtained from all patients and patients' relatives.

\section{Conflict of Interest}

No potential conflict of interest relevant to this article was reported.

\section{References}

1. Atieh MA, Alsabeeha NH, Payne AG, Duncan W, Faggion CM, Esposito M. Interventions for replacing missing teeth: alveolar ridge preservation techniques for dental implant site development. Cochrane Database Syst Rev 2015;(5):CD010176.

2. Camargo PM, Lekovic V, Weinlaender M, Klokkevold PR, Kenney EB, Dimitrijevic B, et al. Influence of bioactive glass on changes in alveolar process dimensions after exodontia. Oral Surg Oral Med Oral Pathol Oral Radiol Endod 2000;90:581-6.

3. Iasella JM, Greenwell H, Miller RL, Hill M, Drisko C, Bohra AA, et al. Ridge preservation with freeze-dried bone allograft and a collagen membrane compared to extraction alone for implant site development: a clinical and histologic study in humans. J Periodontol 2003;74:990-9.

4. Lekovic V, Kenney EB, Weinlaender M, Han T, Klokkevold P, Nedic M, et al. A bone regenerative approach to alveolar ridge maintenance following tooth extraction. Report of 10 cases. J Periodontol 1997;68:563-70.

5. Lekovic V, Camargo PM, Klokkevold PR, Weinlaender M, Kenney EB, Dimitrijevic B, et al. Preservation of alveolar bone in extraction sockets using bioabsorbable membranes. J Periodontol 1998;69:1044-9.

6. Schropp L, Wenzel A, Kostopoulos L, Karring T. Bone healing and soft tissue contour changes following single-tooth extraction: a clinical and radiographic 12-month prospective study. Int J Peri- odontics Restorative Dent 2003;23:313-23.

7. Tan WL, Wong TL, Wong MC, Lang NP. A systematic review of post-extractional alveolar hard and soft tissue dimensional changes in humans. Clin Oral Implants Res 2012;23 Suppl 5:1-21.

8. John V, De Poi R, Blanchard S. Socket preservation as a precursor of future implant placement: review of the literature and case reports. Compend Contin Educ Dent 2007;28:646-53; quiz 654, 671.

9. Ten Heggeler JM, Slot DE, Van der Weijden GA. Effect of socket preservation therapies following tooth extraction in non-molar regions in humans: a systematic review. Clin Oral Implants Res 2011;22:779-88.

10. Hämmerle $\mathrm{CH}$, Araújo MG, Simion M; Osteology Consensus Group 2011. Evidence-based knowledge on the biology and treatment of extraction sockets. Clin Oral Implants Res 2012;23 Suppl 5:80-2.

11. Vignoletti F, Matesanz P, Rodrigo D, Figuero E, Martin C, Sanz M. Surgical protocols for ridge preservation after tooth extraction. A systematic review. Clin Oral Implants Res 2012;23 Suppl 5:22-38.

12. Vittorini Orgeas G, Clementini M, De Risi V, de Sanctis M. Surgical techniques for alveolar socket preservation: a systematic review. Int J Oral Maxillofac Implants 2013;28:1049-61.

13. Damien CJ, Parsons JR. Bone graft and bone graft substitutes: a review of current technology and applications. J Appl Biomater 1991;2:187-208

14. Habal MB. Different forms of bone grafts. In: Habal MB, Reddi AH, Mitchell J, eds. Bone grafts and bone substitutes. Philadelphia: W.B. Saunders; 1992:6-8.

15. Hench LL. Bioceramics: from concept to clinic. J Am Ceram Soc 1991;74:1487-510.

16. Greenwald AS, Boden SD, Goldberg VM, Khan Y, Laurencin CT, Rosier RN; American Academy of Orthopaedic Surgeons. The Committee on Biological Implants. Bone-graft substitutes: facts, fictions, and applications. J Bone Joint Surg Am 2001;83 Suppl 2 Pt 2:98-103.

17. SureshKumar G, Thamizhavel A, Girija EK. Microwave conversion of eggshells into flower-like hydroxyapatite nanostructure for biomedical applications. Mater Lett 2012;76:198-200.

18. SureshKumar G, Thamizhavel A, Yokogawa Y, NarayanaKalkura S, Girija EK. Synthesis, characterization and in vitro studies of zinc and carbonate co-substituted nano-hydroxyapatite for biomedical applications. Mater Chem Phys 2012;134:1127-35.

19. SureshKumar G, Girija EK. Flower-like hydroxyapatite nanostructure obtained from eggshell: a candidate for biomedical applications. Ceram Int 2013;39:8293-9.

20. Park JW, Bae SR, Suh JY, Lee DH, Kim SH, Kim H, et al. Evaluation of bone healing with eggshell-derived bone graft substitutes in rat calvaria: a pilot study. J Biomed Mater Res A 2008;87:203-14.

21. Kim SH, Kim W, Cho JH, Oh NS, Lee MH, Lee SJ. Comparison of bone formation in rabbits using hydroxyapatite and $\beta$-tricalcium phosphate scaffolds fabricated from egg shells. Adv Mater Res 2008;47-50:999-1002.

22. Lee SW, Kim SG, Balázsi C, Chae WS, Lee HO. Comparative study of hydroxyapatite from eggshells and synthetic hydroxyapatite for bone regeneration. Oral Surg Oral Med Oral Pathol Oral Radiol 2012;113:348-55.

23. Kattimani V, Lingamaneni KP, Chakravarthi PS, Kumar TS, Siddharthan A. Eggshell-derived hydroxyapatite: a new era in bone regeneration. J Craniofac Surg 2016;27:112-7.

24. Kattimani VS, Chakravarthi SP, Neelima Devi KN, Sridhar MS, Prasad LK. Comparative evaluation of bovine derived hydroxyapatite and synthetic hydroxyapatite graft in bone regeneration of human maxillary cystic defects: a clinico-radiological study. Indian J Dent Res 2014;25:594-601.

25. Kattimani VS, Bajantai NV, Sriram SK, Sriram RR, Rao VK, Desai PD. Observer strategy and radiographic classification of healing after grafting of cystic defects in maxilla: a radiological appraisal. J Contemp Dent Pract 2013;14:227-32. 
26. Brownfield LA, Weltman RL. Ridge preservation with or without an osteoinductive allograft: a clinical, radiographic, micro-computed tomography, and histologic study evaluating dimensional changes and new bone formation of the alveolar ridge. J Periodontol 2012;83:581-9.

27. Morjaria KR, Wilson R, Palmer RM. Bone healing after tooth extraction with or without an intervention: a systematic review of randomized controlled trials. Clin Implant Dent Relat Res 2014;16:120.

28. Ripamonti U. Osteoinduction in porous hydroxyapatite implanted in heterotopic sites of different animal models. Biomaterials 1996; 17:31-5.

29. Kasaj A, Willershausen B, Reichert C, Röhrig B, Smeets R, Schmidt M. Ability of nanocrystalline hydroxyapatite paste to promote human periodontal ligament cell proliferation. J Oral Sci 2008;50:279-85.

30. Gosain AK, Song L, Riordan P, Amarante MT, Nagy PG, Wilson CR, et al. A 1-year study of osteoinduction in hydroxyapatitederived biomaterials in an adult sheep model: part I. Plast Reconstr Surg 2002;109:619-30.

31. Gross JS. Bone grafting materials for dental applications: a practical guide. Compend Contin Educ Dent 1997;18:1013-8, 1020-2, 1024, passim; quiz.

32. Darby I. Periodontal materials. Aust Dent J 2011;56 Suppl 1:10718.

33. Ashman A. Postextraction ridge preservation using a synthetic alloplast. Implant Dent 2000;9:168-76.

34. Gholami GA, Najafi B, Mashhadiabbas F, Goetz W, Najafi S. Clinical, histologic and histomorphometric evaluation of socket preservation using a synthetic nanocrystalline hydroxyapatite in comparison with a bovine xenograft: a randomized clinical trial. Clin Oral Implants Res 2012;23:1198-204.

35. Götz W, Gerber T, Michel B, Lossdörfer S, Henkel KO, Heinemann F. Immunohistochemical characterization of nanocrystalline hydroxyapatite silica gel (NanoBone(r)) osteogenesis: a study on biopsies from human jaws. Clin Oral Implants Res 2008;19:101626.

36. Chris Arts JJ, Verdonschot N, Schreurs BW, Buma P. The use of a bioresorbable nano-crystalline hydroxyapatite paste in acetabular bone impaction grafting. Biomaterials 2006;27:1110-8.

37. Webster TJ, Ergun C, Doremus RH, Siegel RW, Bizios R. Enhanced functions of osteoblasts on nanophase ceramics. Biomaterials 2000;21:1803-10.

38. Froum S, Orlowski W. Ridge preservation utilizing an alloplast prior to implant placement--clinical and histological case reports.
Pract Periodontics Aesthet Dent 2000;12:393-402; quiz 404.

39. Ashman A, LoPinto J, Rosenlicht J. Ridge augmentation for immediate postextraction implants: eight year retrospective study. Pract Periodontics Aesthet Dent 1995; 7:85-94; quiz 95.

40. Boyne PJ. Use of HTR in tooth extraction sockets to maintain alveolar ridge height and increase concentration of alveolar bone matrix. Gen Dent 1995;43:470-3.

41. Pagni G, Pellegrini G, Giannobile WV, Rasperini G. Postextraction alveolar ridge preservation: biological basis and treatments. Int $\mathrm{J}$ Dent 2012;2012:151030.

42. Fickl S, Zuhr O, Wachtel H, Bolz W, Huerzeler MB. Hard tissue alterations after socket preservation: an experimental study in the beagle dog. Clin Oral Implants Res 2008;19:1111-8.

43. Araújo MG, Lindhe J. Dimensional ridge alterations following tooth extraction. An experimental study in the dog. J Clin Periodontol 2005;32:212-8.

44. Aimetti M, Romano F, Griga FB, Godio L. Clinical and histologic healing of human extraction sockets filled with calcium sulfate. Int J Oral Maxillofac Implants 2009;24:902-9.

45. Murphy KG. Postoperative healing complications associated with Gore-Tex periodontal material. Part II. effect of complications on regeneration. Int J Periodontics Restorative Dent 1995;15:548-61.

46. Murphy KG. Postoperative healing complications associated with Gore-Tex periodontal material. Part I. incidence and characterization. Int J Periodontics Restorative Dent 1995;15:363-75.

47. De Sanctis M, Zucchelli G, Clauser C. Bacterial colonization of bioabsorbable barrier material and periodontal regeneration. J Periodontol 1996;67:1193-200.

48. Miron RJ, Choukroun J. Platelet rich fibrin in regenerative dentistry: biological background and clinical indications. Hoboken: John Wiley \& Sons; 2017.

49. Kattimani VS, Lingamaneni KP. Natural bioceramics: our experience with changing perspectives in the reconstruction of maxillofacial skeleton. J Korean Assoc Oral Maxillofac Surg 2019;45:34-42.

How to cite this article: Kattimani VS, Lingamaneni KP, Kreedapathi GE, Kattappagari KK. Socket preservation using eggshellderived nanohydroxyapatite with platelet-rich fibrin as a barrier membrane: a new technique. J Korean Assoc Oral Maxillofac Surg 2019;45:332-342. https://doi.org/10.5125/jkaoms.2019.45.6.332 